\title{
Optical Signal Processing in Highly-Nonlinear Chalcogenide Planar Waveguides
}

\author{
M. D. Pelusi, V. G. Ta'eed, M. R. E. Lamont, and B. J. Eggleton \\ CUDOS, University of Sydney, NSW 2006, Australia \\ e-mail: m.pelusi@physics.usyd.edu.au
}

S. Madden, D.-Y. Choi, D.A.P. Bulla, and B. Luther-Davies

CUDOS, Laser Physics Centre, The Australian National University, Canberra, ACT 0200, Australia University of Sydney, NSW 2006, Australia

\begin{abstract}
Bit-error rate performance of newly developed $\mathrm{As}_{2} \mathrm{~S}_{3}$ planar waveguides in applications of highspeed all-optical time-division demultiplexing and wavelength conversion of 40-160 Gb/s optical signals through the use of four-wave mixing and cross-phase modulation is investigated.
\end{abstract}

\section{Introduction}

Nonlinear optical phenomena can be applied in a variety of ways to enable a suite of all-optical signal processing functions for future high-speed and high-capacity optical communication networks. These include signal regeneration [1], time-division demultiplexing [2-6], wavelength conversion [7-10], phase conjugated/mid span spectrally-inverted transmission [11], parametric amplification, ultra-fast logic gating, buffering and switching. The all-optical nonlinear approach avoids the need for optical to electronic conversions and can therefore circumvent the bandwidth limitations and prohibitive cost and manufacturing complexity of high-speed opto-electronics. This grows more important as data modulation rates increase beyond current $40 \mathrm{~Gb} / \mathrm{s}$ systems to $160 \mathrm{~Gb} / \mathrm{s}$. Nonlinear signal processing can be performed in various media such as optical fibre [1-3],[8],[9], semiconductor optical amplifiers [5] and periodically poled $\mathrm{LiNbO}_{3}$ (PPLN) [6]. While speed of SOAs is ultimately limited by carrier recovery effects, optical fiber exploiting the $\chi^{(3)}$ nonlinearity through the third-order electric susceptibility that gives rise to the optical Kerr effect is near instantaneous and therefore well suited for high-speed signals. Optical fiber also has advantages of broadband low loss transmission and passive operation, not requiring feedback temperature control or electric biasing. On the other hand, while SOA and PPLN require device length on the order of centimetres, fiber based experiments originally using dispersion-shifted fibre (DSF) with nonlinearity coefficient of $3 \mathrm{~W}^{-1} \mathrm{~km}^{-1}$ required kilometres of length to accumulate sufficient nonlinearity with moderate launch powers [2], which made for bulky devices sensitive to environmental perturbations. Development in late 1990's of highly nonlinear fibres with nonlinearity typically 3-7 times higher than DSF enabled shorter lengths of hundreds of metres [3]. Subsequent development of photonic structured fibres pushed the nonlinearity even higher toward $100 \mathrm{~W}^{-1} \mathrm{~km}^{-1}$ [8]. Advances in non-silica based glass fibers exhibiting much higher $\mathrm{n}_{2}$ coefficient dramatically increased fiber nonlinearity beyond another order of magnitude to $>1000 \mathrm{~W}^{-1} \mathrm{~km}^{-1}$, albeit with high loss, enabling signal processing with remarkably short lengths on the order of $1 \mathrm{~m}$ for both $\mathrm{BiO}_{3}$ [1] and the chalcogenide, As-Se [9] fibers.

Beyond shorter fibre lengths, challenge remains for shrinking optical waveguides from discrete fiber devices onto a planar platform whereupon integration of multi-signal processing functions or parallel optical circuits for processing different wavelength signals simultaneously on a single photonic chip can be envisioned. It's development calls for both ultra-highly nonlinearity and low loss to ensure low operating power. Recent progress in silicon waveguides has demonstrated fabrication of sub-micron dimension photonic circuits i.e. nanowires enabling sufficient nonlinearity to be reached in centimetre lengths, albeit with quite high losses, for both all-optical wavelength conversion [7] and phase conjugation [11] at 10-40 Gb/s. However, silicon suffers significant nonlinear losses from free-carrier absorption and two-photon absorption (TPA) that may impede operation at higher bit rates.

In another approach, fabrication of chalcogenide planar rib waveguides based on $\mathrm{As}_{2} \mathrm{~S}_{3}$ glass has been explored to take advantage of the material's ultra-high $\mathrm{n}_{2}$ coefficient up to three orders of magnitude greater than silica, and low TPA to realize ultra-fast Kerr nonlinearity in compact photonic circuits suited to higher signal bit-rates. CUDOS has recently developed a variety of such waveguides exhibiting nonlinearity coefficient up to $2080 \mathrm{~W}^{-1} \mathrm{~km}^{-1}$, with low polarization dependant and insertion losses. Such low losses have even been achieved in more complex circuits consisting of serpentine shaped rib waveguides as shown in Fig. 1 enabling nonlinearity to accumulate along longer lengths up to $22 \mathrm{~cm}$ in a compact $7 \mathrm{~cm}$ size chip [12]. Such high nonlinearity was achieved with comparatively broad mode area of 5-7 $\mu \mathrm{m}^{2}$ without complexity of nanowire fabrication. This leaves room for further nonlinearity enhancement through the shrinking of waveguides dimensions, which also provides means to tailor chromatic dispersion between normal to anomalous regions. The application of these waveguides to optical time-division demultiplexing [4] and wavelength conversion [10] at high bit-rates through use of four wave mixing (FWM) and cross phase modulation (XPM) respectively was explored for validating the bit-error rate performance. 
The demultiplexing of a $160 \mathrm{~Gb} / \mathrm{s}$ signal into its $10 \mathrm{~Gb} / \mathrm{s}$ tributary channels used a $5 \mathrm{~cm}$ length $\mathrm{As}_{2} \mathrm{~S}_{3}$ planar rib waveguide with mode area of $5.7 \mu \mathrm{m}^{2}$ for nonlinearity coefficient of $2080 \mathrm{~W}^{-1} \mathrm{~km}^{-1}$. A $160 \mathrm{~Gb} / \mathrm{s}$ signal at wavelength $\lambda_{\mathrm{s}}=1560 \mathrm{~nm}$ was combined with a $10 \mathrm{GHz}$ source of $1.5 \mathrm{ps}$ pulses at wavelength $\lambda_{\mathrm{p}}=1550 \mathrm{~nm}$ and launched into the waveguide. By FWM, each pump pulse of peak power $4.4 \mathrm{~W}$ coinciding with the signal pulse generated an idler pulse at new wavelength $\lambda_{\mathrm{i}}=2 \lambda_{\mathrm{p}}-\lambda_{\mathrm{s}}=1540 \mathrm{~nm}$. Fig. 2(a) and (b) compare input and output spectra. The $10 \mathrm{~Gb} / \mathrm{s}$ idler channel was extracted with a band-pass optical filter (BPF) to produce the clear output eye diagrams shown in Fig. 2(c). Bit error rate measurements showed low power penalty less than $1 \mathrm{~dB}$ for all sixteen $10 \mathrm{~Gb} / \mathrm{s}$ channels.

Development of the longer $22 \mathrm{~cm}$ length $\mathrm{As}_{2} \mathrm{~S}_{3}$ waveguide shown in Fig. 1 enabled all-optical wavelength conversion of $40-80 \mathrm{~Gb} / \mathrm{s}$ signals. The input signal was co-launched into the waveguide with a $\mathrm{cw}$ laser tuned to the desired output wavelength. By XPM, the $\mathrm{cw}$ mode was nonlinearly phase modulated in proportion to the instantaneous co-propagating signal power, and the corresponding frequency modulated side-bands where optically filtered to obtain a replica of the input signal at the new wavelength. Fig. 2(d) shows the converted spectra for launching $80 \mathrm{~Gb} / \mathrm{s}$ signal of 1.5 ps pulses at $1550 \mathrm{~nm}$ wavelength with average launch powers of 24 and $22 \mathrm{dBm}$ for signal and $\mathrm{cw}$ respectively. In case of wavelength converting a $40 \mathrm{~Gb} / \mathrm{s}$ input signal of $4.4 \mathrm{ps}$ pulses, a BER power penalty of $\sim 1 \mathrm{~dB}$ was achieved for wavelength conversion from 1535 to either 1555,1560 or $1565 \mathrm{~nm}$.

Extending application of the waveguides to other optical signal processing functions and higher bit-rates will be aided by further reduction of the input peak power requirements through fabrication advances enabling combination of either longer waveguides or smaller optical mode area, or improved materials for enhancing the nonlinearity coefficient, and to some extent, tailoring dispersion - all while maintaining low propagation losses.
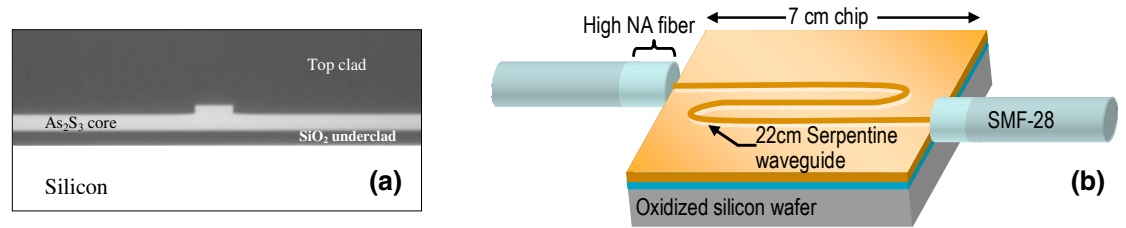

Fig. 1. (a) Optical micrograph of $22 \mathrm{~cm}$ length $\mathrm{As}_{2} \mathrm{~S}_{3}$ rib-waveguide cross-section. (b) Serpentine waveguide geometry

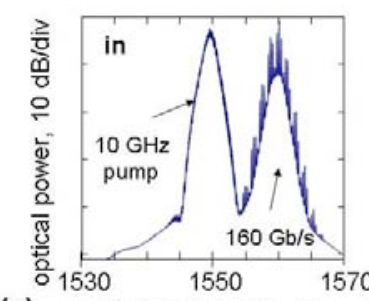

(a) optical wavelength, $\mathrm{nm}$

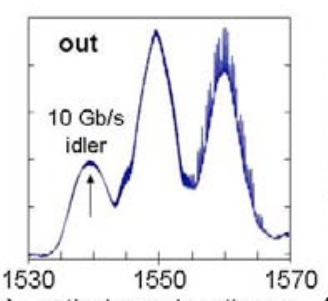

(b) optical wavelength, $\mathrm{nm}$ (c)

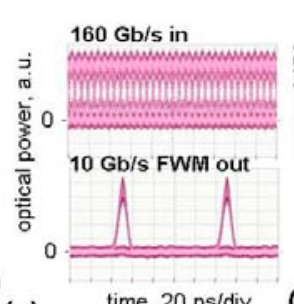

time, $20 \mathrm{ps} / \mathrm{div}$

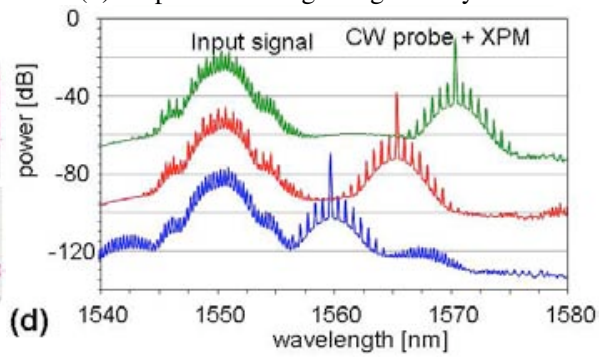

Fig. 2: (a) $160 \mathrm{~Gb} / \mathrm{s}$ demultiplexing: input (b) output spectra and (c) optical eye diagrams. (d) $80 \mathrm{~Gb} / \mathrm{s}$ wavelength conversion output spectra.

\section{References}

[1] F. Parmigiani, S. Asimakis, N. Sugimoto, F. Koizumi, P. Petropoulos and D. J. Richardson, "2R regenerator based on a 2-m-long highly nonlinear bismuth oxide fiber", Opt. Express 14, (2006).

[2] S. Kawanishi, H. Takara, T. Morioka, O. Kamatani and M. Saruwatari, "200 Gbit/s, 100 km time-division-multiplexed optical transmission using supercontinuum pulses with prescaled PLL timing extraction and all-optical demultiplexing", Electron. Lett. 31, (1995).

[3] H. Sotobayashi, C. Sawaguchi, Y. Koyamada, and W. Chujo, "Ultrafast walk-off-free nonlinear optical loop mirror by a simplified configuration for 320-Gbit/s time-division multiplexing signal demultiplexing", Opt. Lett. 27, (2002).

[4] M. D. Pelusi, V. G. Ta'eed, M. R. E. Lamont, S. Madden, D-Y. Choi, B. Luther-Davies, and B. J. Eggleton, "Ultra-high Nonlinear As $\mathrm{S}_{3}$ Planar Waveguide for 160 Gb/s Optical Time-Division Demultiplexing by Four-Wave Mixing" IEEE Photon. Technol. Lett. 19 (2007).

[5] E. Tangdiongga, Y. Liu, H. de Waardt, G.D. Khoe, A.M.J. Koonen, H.J.S. Dorren, X. Shu and I. Bennion, "All-optical demultiplexing of 640 to $40 \mathrm{Gbits} / \mathrm{s}$ using filtered chirp of a semiconductor optical amplifier", Opt. Lett. 32 (2007).

[6] Y. Fukuchi, T. Sakamoto, K. Taira and K. Kikuchi, "All-optical time-division demultiplexing of $160 \mathrm{Gbit} / \mathrm{s}$ signal using cascaded secondorder nonlinear effect in quasi-phase matched LiNbO3 waveguide device", Electron. Lett. 39, (2003).

[7] K. Yamada, H. Fukuda, T. Tsuchizawa, T. Watanabe, T. Shoji, and S. Itabashi, "All-Optical Efficient Wavelength Conversion Using Silicon Photonic Wire Waveguide", IEEE Photon. Technol. Lett. 18, (2006).

[8] J.H. Lee, Z. Yusoff, W. Belardi, M. Ibsen, T. M. Monro, and D. J. Richardson, "A Tunable WDM Wavelength Converter Based on CrossPhase Modulation Effects in Normal Dispersion Holey Fiber", IEEE Photon. Technol. Lett. 15, (2003).

[9] V. G. Ta'eed, L. Fu, M. Pelusi, M. Rochette, I. C. Littler, D. J. Moss, and B. J. Eggleton, "Error free all optical wavelength conversion in highly nonlinear As-Se chalcogenide glass fiber", Opt. Express 14, (2006).

[10] V. Ta'eed, M. Pelusi, B. Eggleton, D.-Y. Choi, S. Madden, Douglas Bulla, Barry Luther-Davies. "Broadband wavelength conversion at 40 $\mathrm{Gb} / \mathrm{s}$ in $\mathrm{As}_{2} \mathrm{~S}_{3}$ planar waveguides" submitted to Optics Express 14, (2006).

[11] S. Ayotte, S. Xu, H. Rong, O. Cohen and M.J. Paniccia "Dispersion compensation by optical phase conjugation in silicon waveguide", Electron. Lett. 43, (2007).

[12] S. Madden, D.-Y. Choi, D. Bulla, A. Rode, B. Luther-Davies, V. G. Ta'eed, M. D. Pelusi, and B. J. Eggleton, "Long, low loss etched As $\mathrm{S}_{3}$ chalcogenide waveguides for all-optical signal regeneration" Opt. Express Submitted (2007). 